\title{
Interactive comment on "Vegetation dynamics and responses to climate anomalies in East Africa" by John Musau et al.
}

\section{Anonymous Referee \#1}

Received and published: 21 February 2018

Overall, from a technical point of view, this work consists of solid data processing and statistical analysis methods for gridded time series. Applied methodologies are well-described and the choices for particular approaches and techniques are generally sufficiently justified, but it does not aim methodological advancement by comparative evaluation of methods, quantitative validation or development of novel techniques. However, from a thematic (environmental) angle, it lacks prior hypotheses on the environmental mechanisms that are underlying to the time series models being tested in the absence of hypotheses the results are presented in a very descriptive way and lack interpretation and synthesis. Only towards the end of the paper, some hints as to the environmental processes at work are suggested and are inductively constructed from sets of positive and negative regression coefficients, where these coefficients are 
treated as data rather than parameters to evaluate and interpret models.

Therefore, the article in its current form does not fully qualify either as a methodological novelty, nor does it present conclusive thematic insights into the role of climate variability in the recent evolution of ecosystems and managed land use systems. I recommend it to be reworked towards either of these directions: A) a systematic evaluation of a new methodology to extract environmental metrics from spatiotemporal data with a thematic case study on East Africa or B) an in-depth thematic study on the variability of climate conditions in East Africa and the mechanistic effects on a different range of ecosystems, modulated by human management. I feel it has most potential for option A, but that would require a stronger emphasis on the novelty of your sequence of extraction methods, and a way to quantitatively evaluate how these methods perform compared to baseline methods or studies.

See attached report for specific comments to the manuscript sections.

Please also note the supplement to this comment:

https://www.earth-syst-dynam-discuss.net/esd-2017-123/esd-2017-123-RC1-

supplement.pdf

Interactive comment on Earth Syst. Dynam. Discuss., https://doi.org/10.5194/esd-2017-123, 2018.

\section{ESDD}

Interactive comment
Printer-friendly version

Discussion paper 